\title{
The microcomputer kit: An excellent small system development tool
}

\author{
DAVID T. KRAUSMAN \\ Department of Psychiatry and Behavioral Sciences, \\ The Johns Hopkins University School of Medicine, Baltimore, Maryland 21205
}

\begin{abstract}
A method is described for demonstrating the integration of a do-it-yourself microcomputer kit into a small dedicated system configuration useful in many clinical and research applications. An Intel SDK-80 microcomputer is used as the main component of an on-line biofeedback system that features a digital display of beat-by-beat heart rate, a continuous feedback of an audio tone proportional to the rate, a CRT display of average rate, and a servo recorder to produce 1 -min bar-graph plots. The compact size, effectiveness, and low cost of the microcomputer permit the development of many small dedicated systems that formerly were impractical applications for expensive and under-utilized minicomputer systems.
\end{abstract}

The use of the minicomputer as an efficient and powerful data processing tool for numerous clinical and research applications has been both recognized and appreciated by the scientific community. As minicomputers have evolved to become the major component of many laboratory facilities, microcomputers are following a similar course at nothing short of an astonishing rate.

The distinguishing characteristic of a microcomputer is one or more large-scale integrated circuit (LSI) elements that perform the basic processing functions. While a minicomputer typically incorporates several hundred low-density integrated circuit (TTL) packages that are distributed over numerous printed circuit boards, the microcomputer generally uses no more than 10 LSi packages integrated into a single circuit board. Small physical size, compactness of components, highly improved reliability, and a superior priceperformance ratio characterize the microcomputer as the apparent successor to the minicomputer in many control, acquisition, and data processing applications.

Since numerous articles have been written on the subject of microcomputers, the reader is referred to current articles by McKenzie (1976), for a detailed description of design and architecture characteristics of the 8080 microcomputer system, and articles by Arnold (1976) and Dessy (1976), for an overview of microcomputer concepts and applications.

Selecting a microprocessor for a specific application presents an unusually difficult task. Software support and hardware guidelines from some manufacturers are virtually nonexistent, and available components and technologies present a perplexing array of choices. Microcomputer configurations range from a collage of hand-wired circuit elements to a complete package system. Currently, the most practical and popular approach to the development of a microcomputer system is the do-it-yourself kit. Priced at $\$ 600$ or less, the microcomputer kit offers an inexpensive and fairly painless way to get a system up and running.

This article describes the integration of a microcomputer kit into a system application useful in many clinical and research laboratories. In particular, the system was designed to demonstrate the basic development techniques that provide for on-line analysis and feedback of heart rate using an Intel SDK-80 microcomputer kit.

\section{THE SDK-80 MICROCOMPUTER KIT}

The SDK -80 is a complete $8080 \mathrm{~A}$ microcomputer system on a single circuit board. The Intel kit costs $\$ 350$ and comes complete with all necessary components and documentation. ${ }^{1}$ Suggested assembly time of the kit was 3 to $5 \mathrm{~h}$, although actual assembly time by the author took $7 \mathrm{~h}$ from the packaged kit to a functioning microcomputer system.

The SDK-80 includes a preprogrammed PROM (programmable read-only memory) system monitor to provide general software utilities and system diagnostics. Communication with the SDK- 80 is initiated through a TTY or CRT console terminal provided by the user. Transmission rate is selectable from 75 to 4,800 baud, and both memory and $\mathrm{I} / \mathrm{O}$ can be easily expanded by simply soldering additional circuit chips into locations provided on the printed circuit board. The circuit board, as illustrated in Figure 1, is about $7 \times 12$ in. overall and features one blank quadrant as a general-purpose wire-wrap pad for user-developed interfacing circuitry.

The SDK-80 system kit includes $2 \mathrm{~K}$ ( $K=1,024$ bytes) of 8708 PROM: The system monitor 


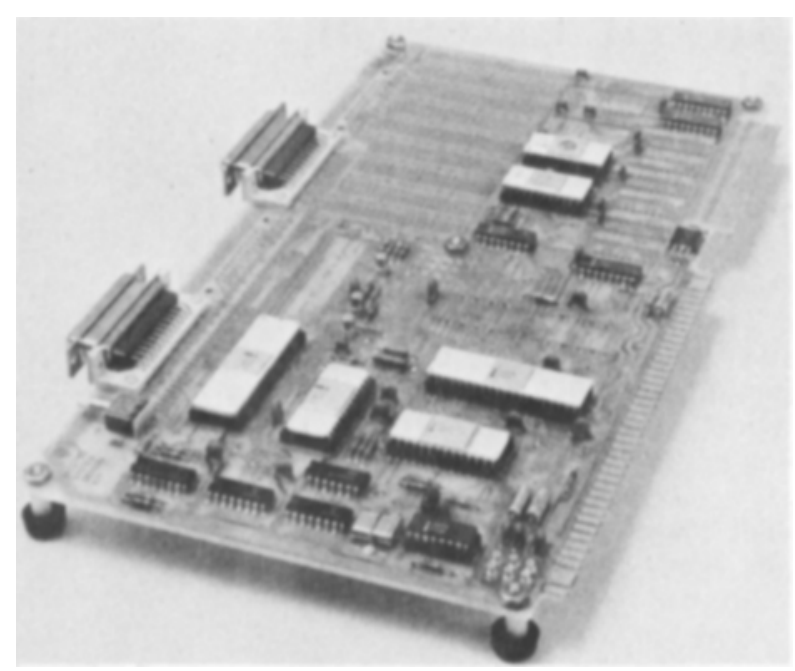

Figure 1. Photo of fully assembled SDK-80 microcomputer kit (courtesy of Intel). Both memory and $\mathrm{I} / \mathrm{O}$ can be easily expanded by simply soldering additional circuit devices into locations provided. A general-purpose wire-wrap pad in the upper right quadrant of the circuit board is provided for custom interfaces. The single board kit is priced at $\$ 350$ and comes complete with all components and documentation necessary to build a high-performance microcomputer system.

requires $1 \mathrm{~K}$ of PROM memory, leaving the other $1 \mathrm{~K}$ available for users' programs. PROM or ROM (read-only memory) is expandable to $4 \mathrm{~K}$ bytes. Two 8111 chips provide 256 bytes of RAM (random access memory, read and write), which is expandable to $1 \mathrm{~K}$ bytes. Input/output devices include a programmable Intel 8255 for 24-line parallel $\mathrm{I} / \mathrm{O}$ and an Intel 8251 USART chip for serial RS-232C or $20-\mathrm{mA}$ current loop I/O for TTY or CRT control. Also provided is a single-level TTL compatible interrupt and a hold line for direct memory access (DMA). A functional block diagram of the internal structure and signal flow of the SDK-80 is shown in Figure 2.

Power for the kit must be provided by the user. Three regulated dc power supplies are required: $5 \mathrm{~V}$ at $1.3 \mathrm{~A}, 12 \mathrm{~V}$ at $.35 \mathrm{~A}$, and $-12 \mathrm{~V}$ at $.20 \mathrm{~A}$.

The SDK-80 monitor is an Intel-developed 8080 program that resides in a preprogrammed PROM. The monitor communicates with the user via an interactive console terminal (TTY or CRT) for implementation of user commands to operate the system. Dialogue between the user and monitor consists of operator keyboard instructions and monitor responses in the form of printed messages. The monitor allows the user to enter, debug, and execute his programs. Facilities for memory modification, register display and modification, and program loading from the console's keyboard are also provided by the permanent monitor program.

Generally, all microcomputer kits, including the SDK-80 require hand assembly for the generation of computer instructions into a computer-compatible machine code. Instruction codes converted to a hexadecimal format are entered into RAM memory one character at a time through the console keyboard. Manual assembly of the instruction codes is not a serious limitation or objection if the user's program is less than $1 \mathrm{~K}$ bytes. However, programming applications that require large amounts of code can rapidly become unwieldy. Long hand-assembled programs that contain errors must be debugged, corrected, and manually reentered in a tedious, time-consuming effort.

RAM memory capacity of the SDK-80 was increased from 256 to 1,024 bytes simply by soldering in six additional 8111 chips at a total cost of about $\$ 30$. This increase easily accommodated the heart rate and utility programs that required about 400 bytes of memory.

There are, of course, many other single-card microcomputer kits which might be more readily available or provide characteristics better suited to the reader's requirements. Some of the more popular alternatives that feature preprogrammed system monitors for program development include: American Microsystems, Inc., Model EVK 399 (\$595); Motorola, Model MEK 6800 (\$149); Fairchild, Model F8 (\$185); and National Semiconductor, Model SC/MP (\$99).

\section{DEVELOPMENT OF THE SYSTEM}

Heart rate biofeedback procedures, although not complex in themselves, provide a straightforward application which permits attention to be focused on the procedure in the development of the microcomputer system and its related peripheral devices. The basic function of the system is to detect heart beats, compute rates, and provide the subject with a digital display of his beat-by-beat heart rate and, simultaneously, continuous audio feedback, in which pitch is proportional to the rate. In addition, a CRT terminal, updated each successive minute, displays the averaged heart rate in beats per minute (BPM), and an on-line servo recorder produces 1-min bar-graph plots.

Figure 3 is a schematic diagram of the heart rate feedback system. The sequential description of the system's operation is as follows: Heart rate information is detected via a Gilson Model T-4020 finger-pulse pickup. This signal is capacitively coupled and amplified in operational amplifier (op amp) A1. A second ac coupled circuit produces a slight differentiation of the pulse signal and is fed to op amp A2, configurated as a comparator or Schmitt trigger circuit. The output signal of the Schmitt (Swinnen, 1968) is shaped and clamped, using a 5-V Zener diode, and coupled to a 10-microsec one-shot multivibrator (IC7). The output of this one-shot directly drives the interrupt line of the SDK-80 microcomputer.

Output lines from the SDK-80's 24-bit I/O (Ports $\mathrm{A}, \mathrm{B}$, and $\mathrm{C}$ ) are programmed accordingly: Port $\mathrm{A}$ ( 8 bits, PA0-PA7) is connected through two line- 


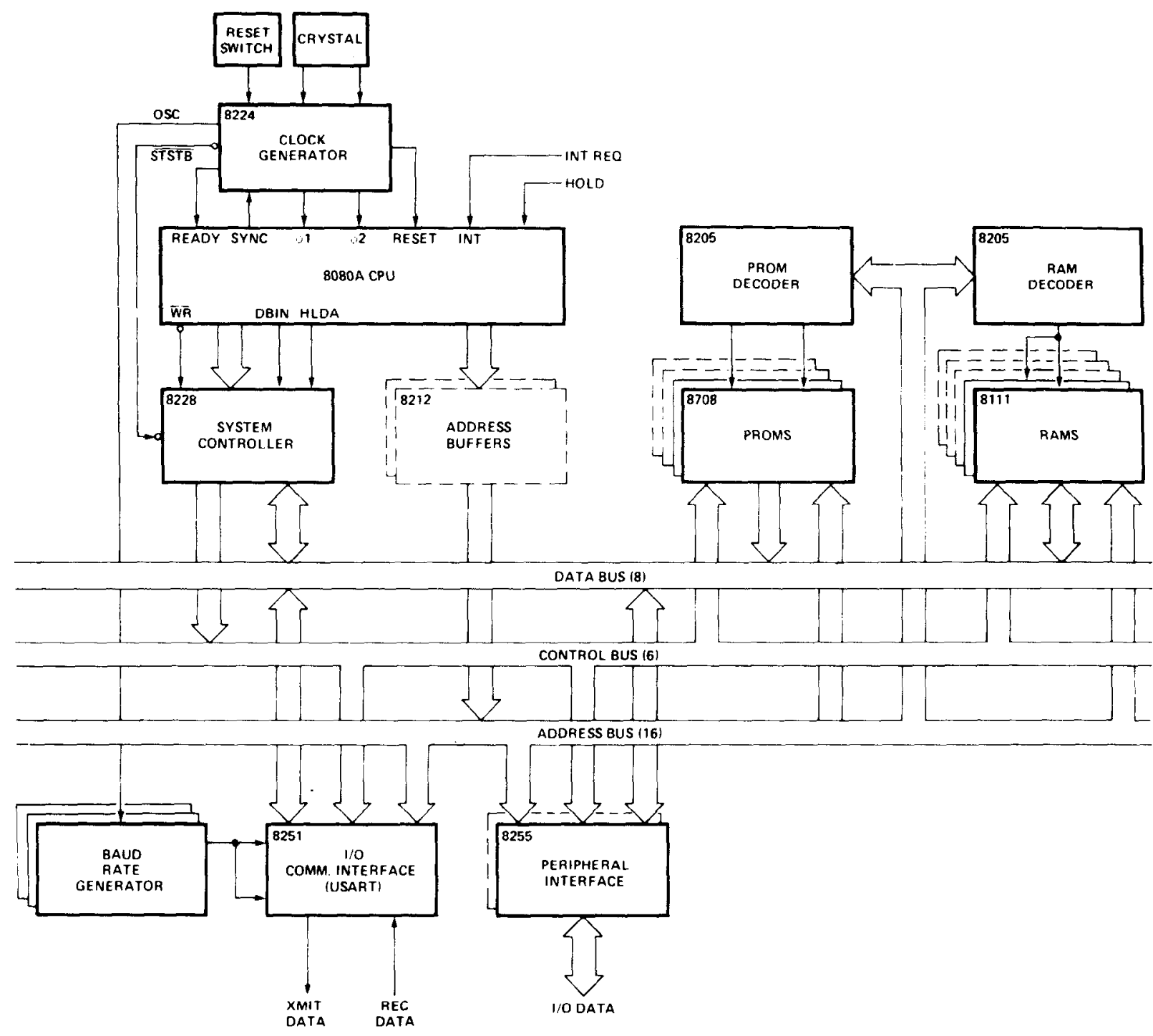

Figure 2. Functional block diagram of the bus structure, signal flow, and circuit organization of the SDK-80 (courtesy of Intel).

buffer chips (IC5, IC6) to two BCD-to-seven-segment decoder/driver chips (IC2, IC3), which in turn drive two LED (light-emitting diodes) displays. These two units represent the mid and least significant digits of the heart rate display. The lower 4 bits of Port $C$ (PC0-PC3) drive the most significant LED digit of the heart rate display via a buffer chip (IC4) and the BCD-to-seven-segment decoder/driver (IC1). The LED display units are continually updated as a beat-by-beat readout of the subject's heart rate.

A 12-bit BCD (binary coded decimal) digital-toanalog converter is also connected across Ports $\mathrm{A}$ and $\mathrm{C}$ via the line buffers to produce an analog voltage proportional to the three-digit heart rate value. This voltage is inverted by op amp A3 and conditioned (gain and zero) by op amp A4 for direct connection through a driver, op amp A5, to a voltage controlled, sine-wave oscillator (VCO), the frequency of which is also proportional to the three-digit heart rate value. For a three- digit heart rate change of 50 to 200 beats $/ \mathrm{min}$, the frequency change of the $\mathrm{VCO}$ is 300 to $3,000 \mathrm{~Hz}$. This signal is then amplified, using a conventional audio amplifier and speaker system, and fed back to the subject as an audible, beat-by-beat tone whose frequency is a direct representation of the heart rate value. A tone-reversing switch (S1) provides an increase in pitch as the rate increases while in the "normal" position and, accordingly, provides a decreasing pitch in the "reverse" position.

A second digital-to-analog converter (DAC) is connected across Output Port B (PB0-PB7) of the microcomputer. This DAC is an 8-bit binary device which converts the binary heart rate value at Port $B$ into a linearly proportional output analog voltage. The analog voltage from the DAC is connected to the signal input of a Heathkit servo recorder modified to accept a remote TTL signal for the start-stop control of the chart drive. At 1-min intervals, the microcomputer 


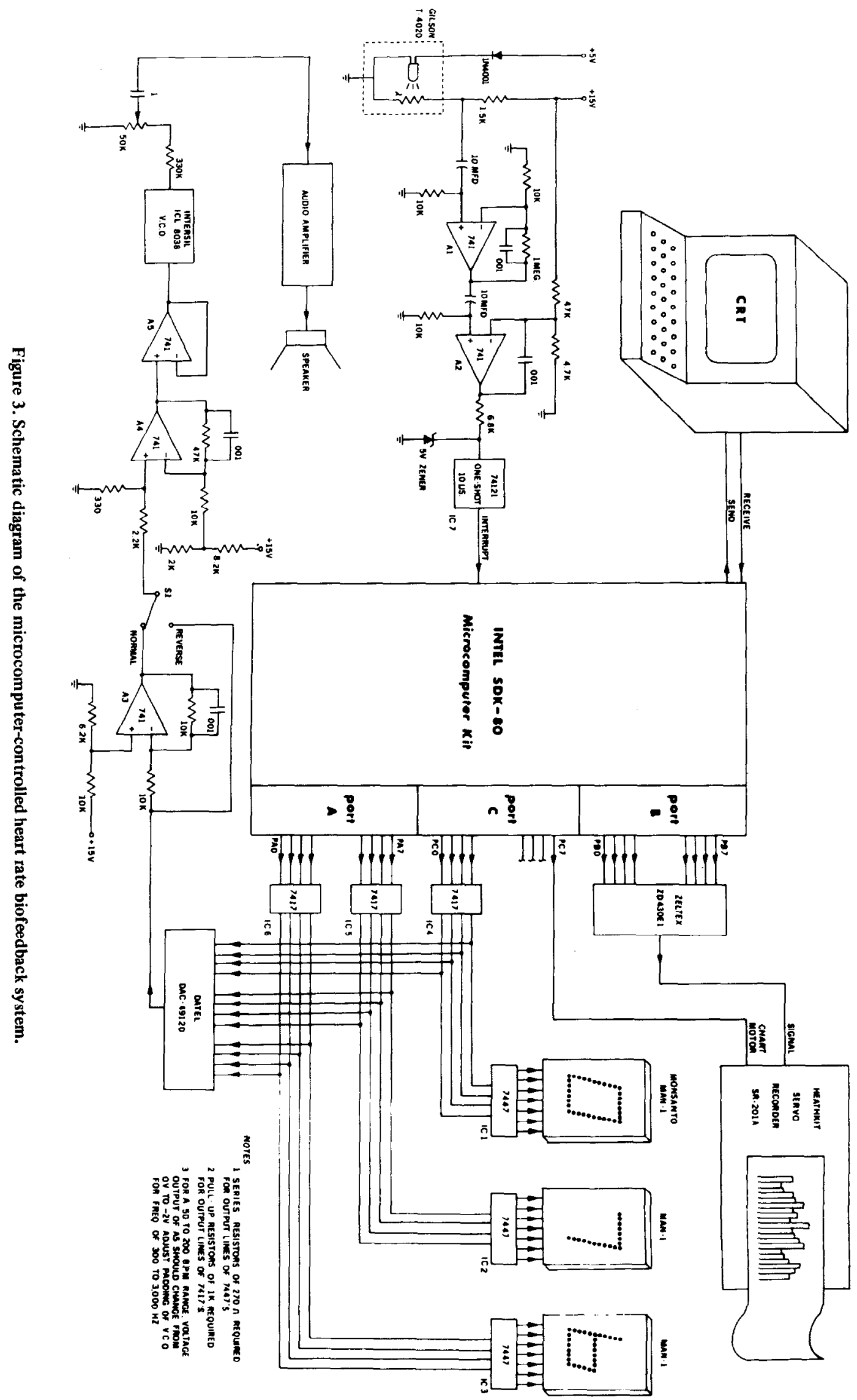


outputs a binary value to Port B which vertically deflects the servo pen to a scaled value corresponding to the averaged 1 -min heart rate value. A motor start signal is also transferred from Port $\mathrm{C}$ Bit 7 of the microcomputer to start the chart motor. After a 4-sec delay, the chart motor stop signal is received from Port $C$ Bit 7; a zero binary value at Port $B$ resets the servo pen to baseline. Repeated 1-min cycling of this procedure produces an on-line graphic display of the subject's average heart rate (Krausman, Baer, \& Anderson, 1974).

The CRT console terminal used to communicate with the SDK-80 microcomputer for program development and debugging is also conveniently used as an on-line logger. Data in a beat-by-beat or averaged format is listed on the CRT screen to provide a real-time visual record of the subject's heart rate changes. Usually the high-speed CRT is an advantage as, for example, the display of real-time data. Although a slower ASR 33 Teletype terminal may not suffice for high-speed displays, it could be equally valuable in some applications for entering various programs into the microcomputer using the paper-tape reader. Accordingly, once the microcomputer application is working, the terminal could be removed altogether, resulting in a less expensive end product.

With the single addition of a second 24-bit Intel 8255 chip (about $\$ 17$ ), the I/O capacity of the SDK-80 microcomputer system can be expanded to facilitate at least six additional LED display digits. Functions such as criterion, reward, and achievement displays could then be easily added and programmed into the feedback system.

The SDK-80 microcomputer system can also be used to extend and enhance the functions of many of the commercial biofeedback systems currently on the market. Most of these units provide auxiliary output jacks for both digital pulses and analog, signals which can interface into the SDK-80 either directly or through a single analog-to-digital converter (ADC) module. On-line data acquisition and analysis of processed functions, such as EMG, EEG, temperature, respiration, etc., derived from these standard biofeedback units is an ideal application for a microcomputer system. Also, utilization of analog preprocessing techniques described by Krausman, Ehrlich, and Brady (1972) can be effectively integrated into the microcomputer system for analysis of basic physiological functions, such as blood pressure and blood flow, which are inherently complex and difficult to process.

\section{PROGRAMMING THE SYSTEM}

The hand-assembled program used to implement the heart rate feedback paradigm as listed in Figure 4 was sequentially loaded into RAM memory of the SDK-80 via the keyboard of the CRT console. Organized into a simple "background-foreground" structure, the program was interspersed throughout RAM locations 1,000 to $1,264 \mathrm{Hex}$. Locations 1,000 through $105 \mathrm{C}$ provide the main background routine for controlling the servo recorder and the 60-sec cycle sequence, with subroutine "timer" (10A0) providing the timing functions and subroutine "CRT" (11A0) providing the CRT display. In general, these background routines are programs that do little processing but principally control $\mathrm{I} / \mathrm{O}$ operations. Foreground is defined to be any program operation executed after receipt of an interrupt command from an external peripheral device. In this immediate application, the heart beat initiates an interrupt request to the microcomputer to perform the foreground "service" routine (1100) for computing the interbeat interval, converting this value into beats/minute, converting the binary beats/minute into a $\mathrm{BCD}$ value for updating the LED displays, and accordingly changing the frequency of the feedback tone. The clock routine for computing the interbeat interval is structured into the background loop (timer routine) using the 1.95 microsec cycle time of the microcomputer itself to generate the millisecond counter. In applications where excessive $\mathrm{I} / \mathrm{O}$ operations of the CRT or other peripheral device require an inordinate amount of background time, an external clock generating 1-msec interrupts to the SDK-80 would be advisable.

Undoubtedly, careful and efficient programming of the microcomputer will be the key factor in developing a successful system. Because of limited memory resources and small word size ( 8 bits), programs must be especially well planned and concise. While microcomputers are less sophisticated and less powerful than today's minicomputers, programming knowledge is directly transferable. However, microcomputer programming is directly dependent on the design of custom interfaces and requires the programmer to possess an additional, comprehensive understanding of the external hardware circuitry being controlled.

Newcomers exposed to the intricacies of small computer systems for the first time will be faced with the usual problems of groping their way through some difficult programming and hardware concepts. An ideal aid in dealing with these new concepts is to attend a training workshop offered by the microcomputer manufacturers. The training program sponsored by Intel covers in detail both hardware and software development. The fee for this 5-day course is $\$ 425$. In addition to the training support, Intel also supports a users' program library. The users' library (membership fee of $\$ 100)$ is a collection of general-purpose programs and routines written by users of the 8080 microcomputer system. This library of programs not only saves the programmer many hours of programming and debugging time, but also serves as an excellent learning tool for those less familiar with assembly language programming. 


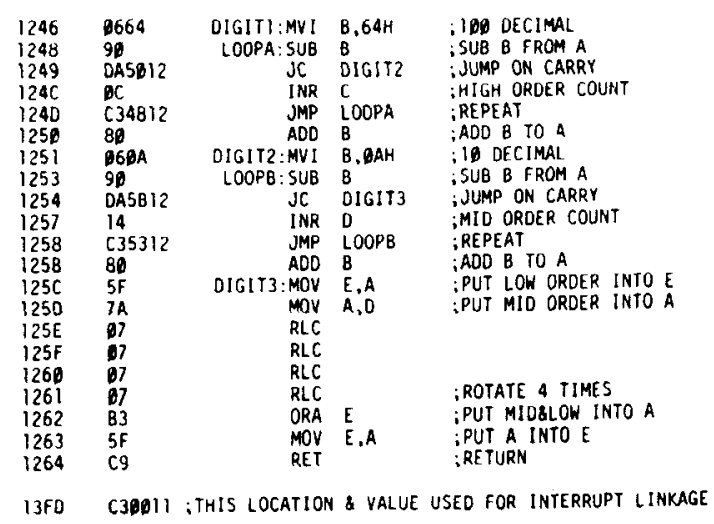

Figure 4. (Continued)

\section{MEMORY CONSIDERATIONS}

Unlike many minicomputers which incorporate magnetic core memory as the standard program storage facility, the SDK-80 and other microcomputer systems use a combination of newer technology RAM and PROM semiconductor memories. RAM memory, which is considerably cheaper than PROM memory, posesses the inherent disadvantage of requiring continuous power for the retention of its program information. Any momentary interruption of power will erase or destroy the program pattern of the RAM, thereby requiring reentry procedures via the CRT keyboard.

Once the program has been successfully debugged for errorless execution of the biofeedback procedures, an optional method to using RAM as the main memory facility might be considered. The same program that was developed in RAM memory can now be permanently programmed into the 8708 PROM chip supplied with the SDK- $80 \mathrm{kit}$. The PROM is a nonvolatile memory device which retains its program information even in a power-off condition. However, the PROM requires a separate external programming unit to "burn-in" the program pattern. Standard PROM programmers are prohibitively expensive (about $\$ 2,000$ ) compared to the cost of the microcomputer. A basic no-frills do-it-yourself PROM programmer kit (Cramerkit Model 2708/2704) that is asynchronously controlled by your own microcomputer has recently been announced and is available from Cramer Electronics. The Cramerkit costs $\$ 130$ and will accommodate the Intel 8708 PROM.

The 8708 PROM provides an additional feature of being completely reprogrammable. This is accomplished by exposing the small quartz window on the PROM memory chip to a special short-wave ultraviolet light for erasure of the old program and then reburning a new program into the chip with the PROM programmer. Ultraviolet erase units are commercially available from inost of the microcomputer distributors at a cost of about $\$ 150$.

This author has successfully implemented the biofeedback procedure into a useful laboratory system by using only the RAM memory facilities of the SDK-80. Power failures or momentary power interruptions generally occur too infrequently to warrant the extra expense of a PROM programmer and UV eraser, especialiy for the initial development and evaluation of your first microcomputer system. However, once the user is satisfied that his program is in a "finalized" form, he can take advantage of the fact that many semiconductor distributors will program PROMs for a nominal charge, generally on the basis of paper tapes or marked sense cards that the user submits. Semiconductor specialists, for example, will program PROMs for a penny a word in addition to the cost of the PROM.

\section{DISCUSSION}

The decision to integrate a microcomputer into a system development application for the first time is an exciting but somewhat difficult experience. The road to microcomputer use will not be an entirely smooth one. The first-time user should be aware of the problems of learning, familiarizing, and developing a good sense of what can be accomplished easily, and what cannot be accomplished at all. Even with these problems in mind, microcomputers are the most attractive, efficient, and cost effective systems for many development applications. Finally, the user should realize that the microcomputer is here to stay, and its utility can provide a new and powerful tool that will significantly enhance his alternative solution to future instrumentation problems.

\section{REFERENCES}

ARnold, J. T. Microprocessor applications: A less sophisticated approach. Science, 1976, 192, 519-523.

Dessy, R. E. Microprocessors? An end user's view. Science, $1976,192,511.518$.

Krausman, D. T., Baer, R. W., \& Anderson, D. E. Graphic bar display of on-line physiological data using a minicomputer, DAC, and conventional servo recorder. Behavior Research Methods \& Instrumentation, 1974, 6, 347-349.

Krausman, D. T., Ehrlich, W., \& Brady, J. V. Preprocessor adapts cardiovascular signals to a minicomputer. Psychophysiology, 1972, 9, 554-563.

McKenziE, K. A structured approach to microcomputer system design. Behavior Research Methods \& Instrumentation. $1976,8,123-128$.

Swinnen, M. E. T. The design of biomedical instrumentation made easy through the use of operational amplifiers. Psychophysiology, 1968, 5, 178-187.

\section{NOTE}

1. The SDK-80 is available from local Intel distributors, including Almac/Stroum, Components Plus, Component Specialties, Cramer, Elmar, Hamilton-Avnet, Industrial Components, Liberty, Pioneer, Scheridan, and L. A. Varah.

(Received for publication June 28, 1976; revision accepted August 12, 1976.) 